\title{
Promises and Failures of Research in Dynamic Service Composition
}

\author{
Fabio Casati
}

\begin{abstract}
This short articles discusses the evolution of research in composition technologies, from workflows to mashups. It emphasizes the failures of composition technologies and makes the case for domain-specific workflows as a possible successful way to leverage composition technologies.
\end{abstract}

\section{Dynamic Workflows}

This short articles discusses the evolution of research in composition technologies, from workflows to mashups. It takes as a starting point the work reported in the paper Adaptive and dynamic service composition in eFlow [1], published in CAiSE 2000. The objective of that paper was to discuss how we can make a service composition more flexible, deciding at deployment or at runtime which concrete service or sets of services should be invoked. We had already realized, back then, that human intervention in this adaptation would be needed, and so the goal of the paper was to discuss how we could simplify the adaptation process, rather than automating it. At that point, web services and the research area of service composition was in its infancy. Web services themselves were a rather new concept, and indeed in that year we had organized the first workshop on Technologies for e-services in conjunction with the VLDB conference. Back then, research in composition technologies was still mostly in the domain of workflow management.

Workflow technology was born in the late 1980s with the promise to automate office procedures and facilitate the coordination and data flow among employees. Composition and workflows - and the related languages and technologies - seemed to make a lot of sense. We have tasks (and often business tasks) which we do need to coordinate and orchestrate to achieve some business value, so why not design a

\footnotetext{
F. Casati $(\bowtie)$

University of Trento, Trento, Italy

e-mail: Fabio.Casati@unitn.it
} 
simple graphical language to help users specify the coordination logic and manage the data exchange among the activities, thereby also avoiding extra work, data entry errors, and allowing accurate work tracking? It seemed like a no brainer and a sure success.

The equivalent of dynamic composition in the workflow space was called dynamic resource selection, consisting of languages, policies, and technologies for defining to which person or role a workflow task should be assigned. Research in this area has been extensive, and produced many languages that allowed the specification of complex assignment policies that consider aspects such as workers' skills, schedule, and even compliance rules such as separation of duties [3].

However, the success of workflow systems has been much lower than anticipated. Nearly all major software companies and many startups had an offering in the workflow space, but sales were very low. As I reported in my book [2], part of the problem was that workflow systems were very expensive and "heavy", that is, complex to install and operate as they included lots of functionality (this was not the only problem, but we'll get back to this a bit later). Most of the deployments were done for the purpose of tracking work more so than automating it. The implication is that the abundant research results were in fact rarely used and had very little impact in practice, especially research in dynamic resource assignment. In a way, we as researchers were trying to solve an advanced and complex problem without addressing (or even understanding) the basic issues that impeded the success of workflow technology.

\section{Dynamic Service Composition}

In the mid and late 1990s, Enterprise Application Integration (EAI) platforms came about, facilitating the interconnection of enterprise systems. Workflows then evolved from orchestrating the work among humans to orchestrating the information flow across enterprise systems. While EAI platforms were rather successful, workflows still enjoyed limited adoption.

Web services seemed to be a key enabler for composition technologies, overcoming some of the issues that impeded the adoption of workflow systems. What web services and the related middlware bring to the table is (at least in principle) a standard way for describing interfaces and interaction protocols and for transporting data. The thought shared by many researchers and practitioners in the area was that every company would expose their services as web services, following a same standard and publishing their interfaces and protocols, so that it would therefore be easy to write program that access these services. As a result we would have a myriad of services available and with them the opportunity of combining such services to obtain a functionality of interest. A global registry (UDDI[4], you may remember it) would contain pointers to all specifications of all services in the world. 
The main point of attraction here was that because of the highly standardized (at least technically) nature of web services, it would be easy to interoperate with them. If we could develop a (graphical) language and a model to enable the composition of services (with a supporting execution engine), we would have a powerful tool to quickly create complex services out of other services. The high degree of standardization would reduce the complexity of the composition engines, thereby making the products lighter and cheaper.

This technological development fueled research in workflows under the hat of service composition. New (or, actually, refurbished) models and languages started to appear to support the specification of various aspects of a composition.

Despite the hopes, however, the situation soon mirrored what happened to workflows: lots of hype on service composition, lots of tools by big and small companies, lots of investment, and very limited adoption. Part of the problem here has nothing to do with service composition: web services themselves had a lot of success as a concept (more and more functionality is now accessible programmatically) but there is no standard really used for interface and protocol description, it all really boils down to http. To a large extent, interfaces are specified in plain English, and structured in arbitrary ways. Formal or semantic specifications of interfaces is something that requires highly skilled (and very careful, very precise) people. It works in theory but not in practice.

A part of the problem however does have to do with service composition. The issue here is that, while most languages claimed to be "easy to use", in practice service composition required professional programmers. And because programmers knew how to program, it was and is just easier to code the composition with a conventional programming language rather than learn yet another language and install and maintain yet another piece of software. Most programmers also prefer to script rather than to use graphical languages. Composition systems did provide some extra features (such as advanced transactional models) but once again programmers resorted to the comfort of databases, which were well known and widely tested, and in most cases that was enough.

In this context, once again research on dynamic service composition was flourishing, and still today we often come across new papers proposing some algorithms that improves the status quo. Most papers assumed the existence of several services with the same interface and similar functionality, and that consequently these services could be easily and seamlessly swapped. An algorithm would then determine which service provided the best quality at the lower cost, based on various criteria. The main flaw I see now in this thread of research is that (i) swapping a service with another is not easy from a business perspective. Service relationships are based on contractual agreements and in general the service provider a company chooses depends on many factors difficult to encode; (ii) Most importantly, even if two services have the same interface, the hope to replace one with another and have the composition continue to work smoothly is vain. This is because interoperability with any non-trivial service must be repeatedly tested and the meaning of the different parameters must be fully understood. In my experience, this is true no matter how well the service interface is described. Different 
services with the same interface would always behave differently because different implementations interpret and understand parameters differently. The reason why these issues are rarely addressed in papers is that they show up in real settings, but not in lab tests. Composition, including dynamic composition, is intuitively attractive, but it is only when we apply it in a real setting that we see the real issues surfacing. And because there were very few real services out there, it was difficult to have them surface.

Additional thread of research included semantic composition (where algorithms were even trying to understand the service goals and behaviors, going beyond the interface, and take dynamic composition decisions based on that) and goal-oriented planning, where a partial or complete composition is created (semi-)automatically based on a description of the goal of the composition. These approaches suffered and still suffer from issues similar to the one described above, only extended because there are more aspects considered by the algorithms besides the interface.

\section{Lessons Learned}

So, as we saw, history has been repeating in the field of (dynamic) composition. Interestingly, the same is happening today with mashups.

The interesting aspect is, however, to look at those cases where composition is indeed successful. These cases always have a common aspect: they tackle domain-specific problems. Prominent examples of these are data transformation tools or scientific workflows, but there are many others. These tools are specific enough and limited enough to actually become usable by non-programmers and to provide out of the box features that make them attractive. It is the specificity (and the consequent simplicity), not the generality, that makes these tools useful. Somehow we tend as researchers to drive towards generic solution as we feel that this is the goal of research, but in composition technologies we have learned that flexibility comes at a very high price, that of making the technology difficult to use and, very often, useless. The challenge that lies in front of us is, therefore, that of finding ways to easily create domain-specific composition models that are simple enough (and, necessarily, limited enough) to be applicable to one class of problems and that are amenable to non-programmers, meaning, such that non-programmers can create a complete application from start to finish without the need of writing code outside of the platform, or of specifying composition logic that, although graphical, presents all the difficulties (complex parallelism, data transformation, complex exception handling) of programming languages. Unless we can get empower users at the skill level of the average Excel user to write complete composition (possibly after some training), this technology will remain attractive in theory but ineffective in practice.

Acknowledgements A big thanks to the friends, colleagues, and co-authors of the original CAiSE paper: Ski Ilnicki, LiJie Jin, Vasudev Krishnamoorthy, and Ming-Chien Shan. 


\section{References}

1. F. Casati, S. Ilnicki, L.J. Jin, V. Krishnamoorthy and M.C. Shan, Adaptive and Dynamic Service Composition in eFlow, Procs of Caise'00, Sweden. Springer.

2. G. Alonso, F. Casati, H. Kuno, and V. Machiraju. Web Services Concepts, Architectures and Applications. Springer-Verlag, Berlin, 2004.

3. R. Botha and J.Eloff, Separation of duties for access control enforcement in workflow environments, IBM Systems Journal 40 (3), 2001.

4. Luc Clement, Andrew Hately, Claus von Riegen, and Tony Rogers. UDDI Version 3. OASIS UDDI Spec TC, 2004. 